\title{
¿La primera orfebrería del nordeste de la Península Ibérica? Nuevas aportaciones a partir de la cuenta áurea de Cau del Tossal Gros (Torroella del Montgrí, Baix Empordà, Girona)
}

\author{
The earliest metallurgy in the northeast Iberian Peninsula? New analysis of a gold bead \\ from Tossal Gros cave (Torroella de Montgrí, Girona)
}

\author{
Ignacio Soriano Llopis (*) \\ Joaquim Soler Subils (**) \\ Narcís Soler Masferrer (**)
}

\begin{abstract}
RESUMEN
La cuenta bitroncocónica de oro localizada en la cueva sepulcral de Cau del Tossal Gros (Torroella de Montgrí, Baix Empordà, Girona) constituye la primera de este tipo conocida en la Península Ibérica. Estas cuentas son comunes en yacimientos del sur de Francia y se fechan de forma relativa en el Neolítico Final. Con el objetivo de establecer su proceso de producción se han realizado análisis de composición, radiológicos y traceológicos. Los resultados muestran la gran complejidad tecnológica de la pieza, sin paralelos conocidos en la Prehistoria peninsular. La datación radiocarbónica del yacimiento mediante tres fechas AMS y los materiales recuperados confirman una cronología de finales del IV-inicios del III milenio cal ANE. Finalmente, la comparación con los datos disponibles sobre la primera metalurgia del nordeste de la Península Ibérica permite proponer el posible origen, uso y valor social de esta peculiar cuenta áurea.
\end{abstract}

\begin{abstract}
The find of a gold biconvex bead from Tossal Gros burial cave (Torroella de Montgri, Girona) represents the first example of this type known in the Iberian Peninsula. These beads are common in the south of France and are dated to the Late Neolithic. With the aim of establishing
\end{abstract}

(*) Grupo de Investigación Arqueológica del Nordeste Peninsular -GRANEP-y Dpto. de Prehistoria, Facultad de Filosofía y Letras, Universidad Autónoma de Barcelona. Edificio B. 08193 Bellaterra. Correo e.: nachsoriano@hotmail.com

(**) Instituto de Investigación Histórica, Facultad de Letras, Universidad de Girona. Plaça Ferrater i Mora 1. 17071 Girona. Correos e.: joaquim.soler@udg.edu; narcis.soler@udg.edu

Recibido: 9-II-2011; aceptado: 16-IV-2011 their production process, we applied SEM-EDS, radiology and use-wear analysis. The results show the high technological complexity of the bead, which is unparalleled in prehistoric Iberia. The radiocarbon dating of the site by three AMS dates and associated materials confirm a date to the end of $4^{\text {th }}$ - beginning of $3^{\text {rd }}$ millennia cal $B C$. Comparisons with the available data concerning the earliest Northeast Iberian metallurgy allows an assessment of the possible origin, use and social value of this particular golden bead.

Palabras clave: Orfebrería prehistórica; Radiología; MEB-EDX; Traceología metálica; Arqueometalurgia; Dataciones AMS; Prehistoria Reciente; Mediterráneo occidental.

Key words: Prehistoric goldwork; Radiology; SEM-EDS; Metal use-wear analysis; Archaeometallurgy; Late Prehistory; Western Mediterranean.

\section{CONTEXTO AQUEOLÓGICO: LA NECRÓPOLIS DE CAU DEL TOSSAL GROS}

El Tossal Gros es una pequeña elevación en el reborde meridional del macizo del Montgrí (Fig. 1). Este macizo, formado por calcáreas cretácicas que cabalgan sobre materiales terciarios, destaca junto a la costa gerundense y separa el Baix Empordà del Alt Empordà. Se trata de una zona muy carstificada y muchas de sus cavidades, o cau, tienen interés arqueológico. Las más conocidas, el Cau del Duc de Torroella de Montgrí y el Cau del Duc d'Ullà, fueron ocupadas durante el Paleolítico Inferior. Las demás fueron usadas con finalidad se- 


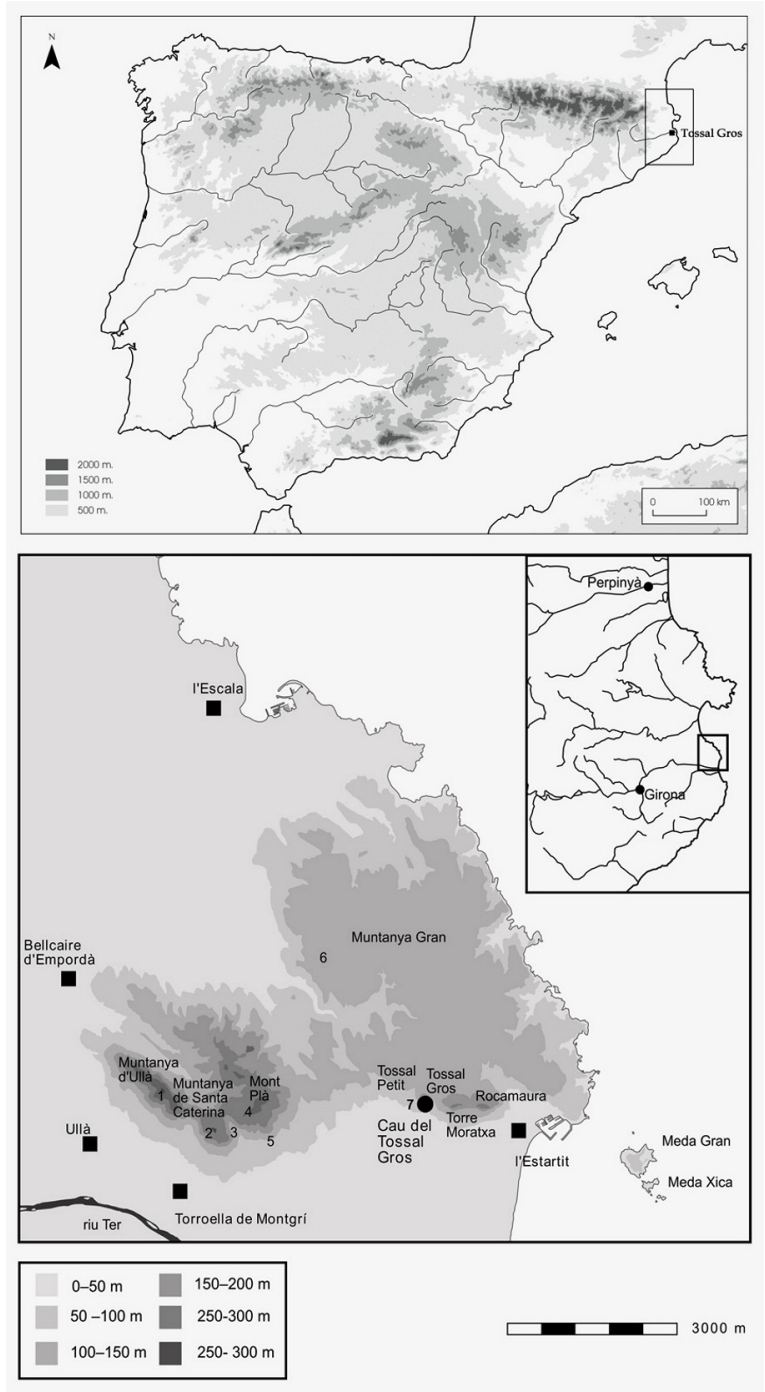

Fig. 1. Localización geográfica del Cau del Tossal Gros (n. ${ }^{\circ}$ 7) (Torroella de Montgrí, Baix Empordà, Girona) en la Península Ibérica. Distribución en el macizo del Montgrí de Oeste a Este de las cavidades citadas en el texto con hallazgos prehistóricos: 1. Cau del Duc d'Ullà; 2. Cau del Duc de Torroella de Montgrí; 3. Cau d'en Calvet; 4. Cau de les Dents; 5. Cau de l'Olivar d'en Maragall; 6. Cau dels Ossos.

pulcral a finales del IV-III milenio cal ANE y su registro arqueológico suele ser escaso o mal conservado. Como excepciones cabe citar el Cau del Ossos (Pascual 1883), que desgraciadamente fue vaciado ya en el siglo XIX, y el Cau d'en Calvet que pudo ser excavado en mejores condiciones y proporcionó numerosos restos humanos y un rico ajuar (Toledo i Mur et al. 1992).
El Cau del Tossal Gros es una cavidad conocida de antaño, localizada en una sima situada en lo más alto del Tossal Gros (161 m), desde donde se goza de impresionantes vistas sobre el llano del bajo Ter y el Baix Empordà. Una grieta en el suelo da paso a un acceso estrecho y de fuerte pendiente, de más de $2 \mathrm{~m}$ de altura, que conduce a una sala de unos $16 \mathrm{~m}$ de largo por 3 o $5 \mathrm{~m}$ de ancho según los lugares. Esta cavidad se rellenó parcialmente con tierra y piedras del exterior. Hay también grandes bloques desprendidos que proceden de sus paredes. La formación de espeleotemas es escasa. A partir de esta sala se abren galerías pequeñas e inaccesibles, casi totalmente colmatadas por sedimento y bloques removidos y acumulados durante las intervenciones que ha sufrido el lugar. Un sector con sedimento hundido, succionado hacia el fondo, sugiere probables cavidades inferiores y el carácter de sima del lugar. En época prehistórica la sala fue usada como enterramiento y por ello su entrada exterior fue modificada para hacerla más monumental, añadiéndole un corredor de losas rodeado de un túmulo (Pericot y Esteva 1973).

Desde la primera mitad del siglo XX el yacimiento ha sido objeto de intervenciones arqueológicas cortas y esporádicas. Las primeras se deben a L. Pericot, originario de Torroella de Montgrí, quien siendo estudiante emprendió en varios de los caus del Montgrí más bien visitas que campañas de excavación regulares y de larga duración. Sin embargo, los resultados fueron significativos y los referentes a las cavidades sepulcrales quedaron reflejados en su tesis doctoral sobre el megalitismo en Catalunya (Pericot 1925, 1950) y en otras publicaciones (Pericot 1939, 1960). En 1923 encontró en el Cau del Tossal Gros un gran cuchillo de sílex y huesos humanos y en 1925 lo volvió a visitar con su profesor P. Bosch Gimpera y otros acompañantes (Pericot 1986).

Posteriormente, el yacimiento sufrió muchas remociones, de las que apenas se conserva documentación (Vert 1980). Durante una visita en 1996 dos de nosotros (J. S. y N. S.) recogimos en superficie una gran lámina de sílex. Ante la evidencia de que el lugar todavía podía proporcionar material prehistórico, en 1998 y 1999 limpiamos la cavidad y tamizamos las tierras removidas. Estas intervenciones se insertaban en un proyecto de estudio del sepulcro y de restauración de su entrada megalítica, acordado entre la Universidad de Girona y el Museo del Montgrí y del Bajo Ter de 
Torroella de Montgrí, hoy Museo del Mediterráneo. Constatamos que las antiguas excavaciones no habían llegado a todas partes (Soler et al. 2002). En 1998, trabajando junto a la pared norte de la sala mayor, a medida que la acumulación de sedimento descendía y la pared de la cavidad se retiraba, llegamos a un espacio de apenas un palmo de altura, hasta aquel momento inaccesible. Este se caracterizaba por una gran abundancia de fragmentos de huesos y de dientes humanos en superficie, que a causa de la pendiente rodaron a aquel rincón. Aquí recogimos la cuenta de oro estudiada en este trabajo, limpia y brillante y sin que siquiera el orificio interior estuviera taponado.

El material arqueológico procedente del Cau del Tossal Gros es limitado pero significativo. Según L. Pericot $(1925,1939,1950)$ en sus visitas de 1923 y 1925 pudo recoger huesos humanos de diferentes individuos, un cuchillo o gran lámina de sílex, una punta bifacial de sílex blancuzco de $3 \mathrm{~cm}$ de longitud con pedúnculo incipiente, un punzón de hueso de $10,5 \mathrm{~cm}$ de longitud, un incisivo de jabalí pulido, fragmentos de cerámica a mano tosca sin decorar y una pequeña hacha de piedra negra, muy bien pulimentada, de $3,5 \mathrm{~cm}$ de longitud (Fig. 2B). Todo este material fue depositado en el Museo Arqueológico de Catalunya (Pericot 1939: 123-124; 1950: 93).

Las rebuscas del Centro de Estudios del Montgrí también proporcionaron un hacha pulimentada pequeña de traquidolerita, un pendiente de hueso, fragmentos de cerámica prehistórica y un buen número de restos humanos, sobre todo dientes (Vert 1980).

Entre los hallazgos arqueológicos de 1996, 1998 y 1999 hay que destacar (Fig. 2A) una gran lámina de sílex de $220 \times 20 \times 6 \mathrm{~mm}$, una punta foliácea bifacial de sílex gris de origen languedociense $(54 \times 19 \times 7 \mathrm{~mm})$, como las que aparecen con frecuencia en los yacimientos sepulcrales del III milenio cal ANE, una pequeña hacha de piedra pulimentada $(33 \times 20 \times 7 \mathrm{~mm})$ de forma trapezoidal, tres cuentas de piedra cilíndricas de unos $12 \mathrm{~mm}$ de diámetro y $4 \mathrm{~mm}$ de grueso, pequeños fragmentos de cerámica prehistórica no decorados, a excepción de dos campaniformes, y finalmente la cuenta de oro que se describirá a continuación. Los abundantes restos de conejo (271) y los escasos de ovicápridos (31), aves (23), peces (13) y un único resto de erizo no se encuentran quemados ni presentan señales antrópicas.
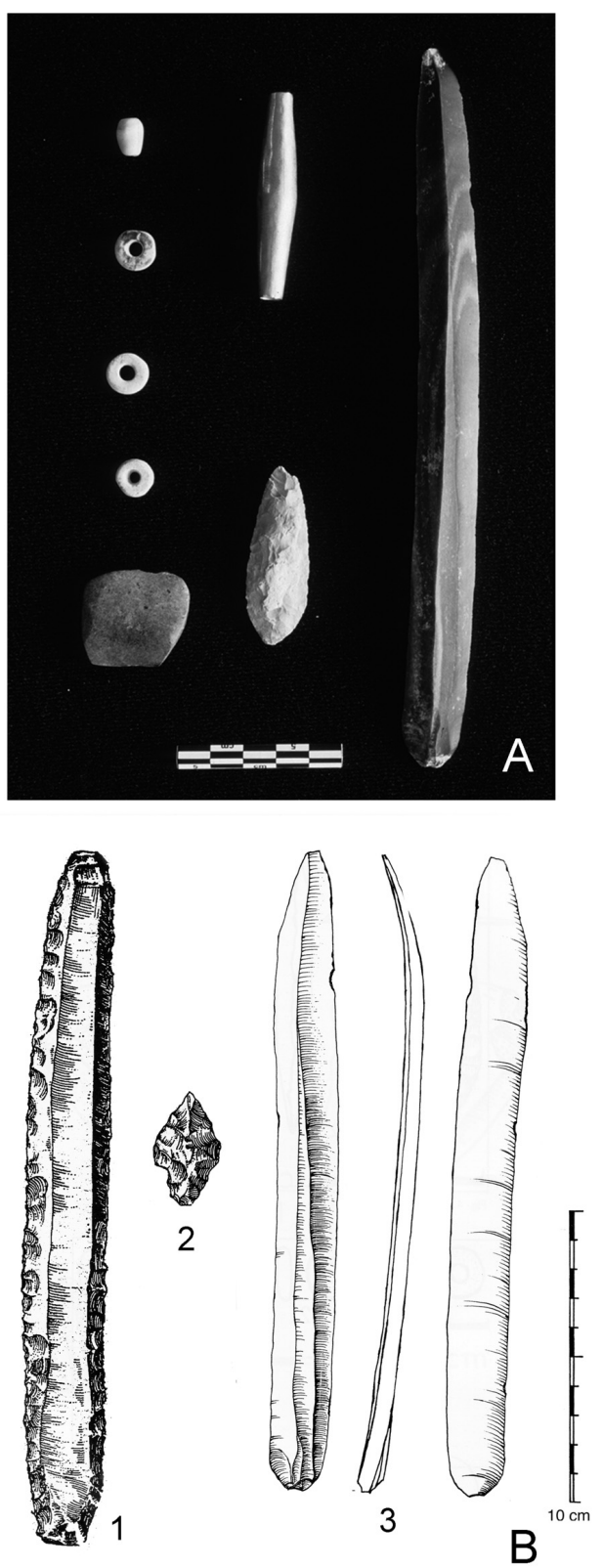

Fig. 2. Cau del Tossal Gros (Torroella de Montgrí, Baix Empordà, Girona). A. Selección de materiales recuperados durante las campañas de 1998 y 1999 (fotografía Narcís Soler). B. Grandes láminas y punta bifacial de sílex procedentes de las excavaciones de Lluís Pericot (1 y 2) (según Pericot 1939: figs. 6 y 7) y de la Universidad de Girona y el Museo del Montgrí y del Bajo Ter (3) (según Soler et al. 2002: fig. 3).

El mayor número de restos corresponde a fragmentos de huesos humanos. Han sido estudiados más de 5.100 entre los conservados en el 
Museo del Mediterráneo y los hallados en 1998 y 1999. Entre ellos, 3.450 son indeterminables y entre los determinables destacan por su número los huesos pequeños de manos y pies así como 379 dientes. Estos últimos han permitido a B. Agustí y J. Fiego (2002) dar un número mínimo de 31 individuos: 20 adultos, 3 adolescentes y 8 niños. Al parecer, fueron depositados sobre el suelo de la cavidad sin ser cubiertos.

\section{LA CUENTA ÁUREA. DESCRIPCIÓN Y CARACTERÍSTICAS}

La cuenta de oro de Cau del Tossal Gros constituye la única de sus características conocida hasta el momento en la Península Ibérica. Este ornamento se ha clasificado según la tipología de Eluère (1977, 1982), la cual se basa en el sistema de suspensión y en su posible funcionalidad. Las cuentas se definen como objetos de suspensión indirecta con un orificio longitudinal para su engarce. En este caso el orificio es central y la morfología bitroncocónica. La pieza se encuentra en perfecto estado de conservación, a pesar de unas pequeñas fisuras alrededor de los orificios. Sus dimensiones (en $\mathrm{mm}$ ) son 61,6 de longitud, 12,3 de anchura máxima, 0,3 de espesor y 6,9 de diámetro en cada orificio (Fig. 3). Tiene un peso de 11,18 gr y actualmente se encuentra depositada en el Museo del Mediterráneo de Torroella de Montgrí (n. ${ }^{\circ}$ de inventario 1299).

La funcionalidad de las cuentas puede ser muy diversa: piezas de collar, pulsera, diadema u objetos similares, junto a cuentas de igual o diferente materia y morfología. En el dolmen de Grah Niol (Arzon, Morbihan, Bretagne), tres cuentas tubulares de oro se asociaban a otras de variscita, aparentemente en un mismo conjunto (Eluère 1977: 393). Pueden igualmente haber constituido complementos de casi cualquier tipo de objeto, unidos con una cuerda o hilo sobre vestimentas, zurrones, mangos de útiles y armas, etc. Por último, pudieron formar parte de elementos de tocado, incrementando la vistosidad que de por sí ya tiene el propio metal. Recientemente se ha documentado dicho uso en el asentamiento campaniforme de Camino de las Yeseras (San Fernando de Henares, Madrid). En una cámara con pozo de acceso se localizó un único individuo masculino joven con 22 cuentas tubulares y 2 láminas perforadas de oro agrupadas alrededor del cráneo.

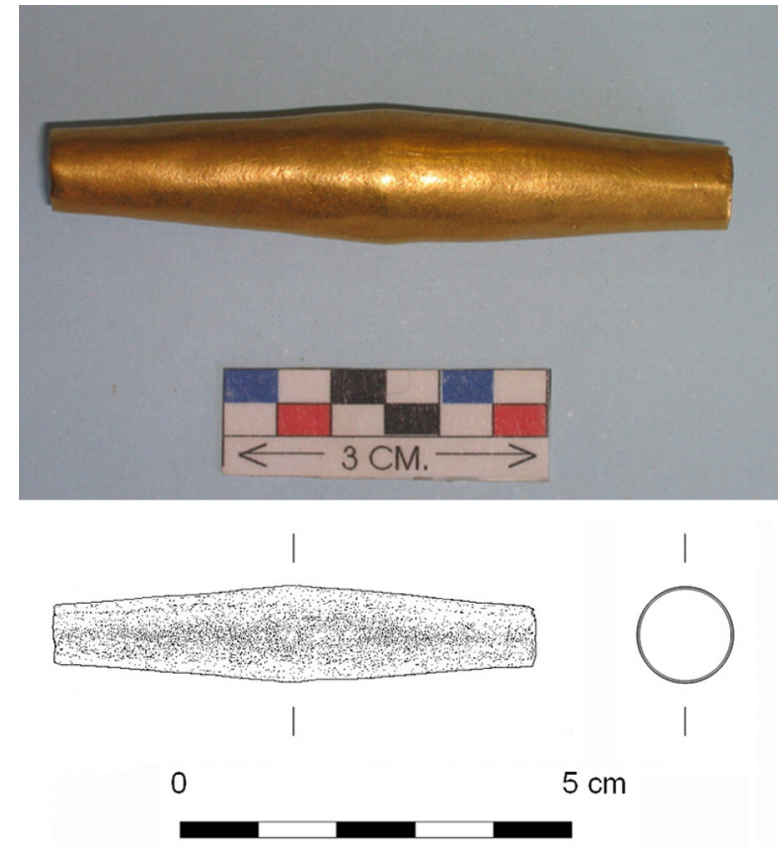

Fig. 3. Cau del Tossal Gros (Torroella de Montgrí, Baix Empordà, Girona): cuenta de oro (dibujo Emiliano Hinojo, fotografía Ignacio Soriano).

Esta asociación ha servido para su interpretación como componentes de una diadema (Blasco y Ríos 2010: 367-368). La documentación de las cuentas in situ es fundamental para proponer su funcionalidad, aunque en muchos casos carecemos de ella. En la cuenta de Cau del Tossal Gros, la ausencia de huellas diagnósticas visibles mediante el estudio traceológico aumenta la dificultad de discernir entre los posibles usos citados (véase más adelante).

\section{ESTUDIO TECNOLÓGICO Y PROCESO DE PRODUCCIÓN}

Uno de los objetivos de este estudio era establecer el proceso seguido para la obtención de la cuenta áurea. Para ello se aplicaron técnicas analíticas como el análisis de la composición, el estudio radiológico y la traceología metálica. En el primer caso se empleó la microscopía electrónica de barrido con dispersión de energías de rayos X (MEB-EDX), mediante un microanalizador QUANTAX Bruker AXS Microanalysis $\mathrm{GmbH}$ del Laboratorio de Microscopía Electróni- 
ca y Microanálisis del Centro de Ciencias Humanas y Sociales (CSIC, Madrid). El escaso espesor de la pieza aseguraba su correcta caracterización con esta técnica de análisis superficial. Además para contrarrestar posibles discrepancias se efectuaron 5 tomas en diferentes zonas del objeto. Los resultados obtenidos ya han sido publicados (Perea et al. 2010: 67-68). Mediante un equipo RX de potencial constante y filmación inherente se radiografió la pieza en distintas posiciones para examinarla en su totalidad. El equipo pertenece a la Asociación Española de Ensayos No Destructivos (AEND, Madrid) y sus características son las siguientes: $1 \mathrm{~mm} \mathrm{Be;} \mathrm{T.F.} 3 \mathrm{~mm}$; distancia foco-película $700 \mathrm{~mm}$; kV 140; exposición a $5 \mathrm{~mA}$ /minuto; película AGFA D7 VACU$\mathrm{PAL} \mathrm{Pb} 0.027$ anterior y posterior. El procesado ha sido manual a $\mathrm{R} 4 \min 20^{\circ} \mathrm{C}, \mathrm{BP} 1 \min 18^{\circ} \mathrm{C}$, F $10 \mathrm{~min} 18^{\circ} \mathrm{C}$ y L $20 \mathrm{~min}$. En el análisis traceológico se utilizó el citado microscopio electrónico y un microscopio óptico de luz reflejada Olympus BX-51 con objetivos de 50 a 500X, del Servicio de Análisis Arqueológicos de la Universidad Autónoma de Barcelona.

Los datos de la composición muestran que el oro es de gran pureza, conteniendo una cantidad muy baja de plata (menos del 2\%) (Tab. 1). Este elemento, al igual que el cobre, es común en el oro nativo de tipo aluvial. Como no existen demasiados análisis de oro aluvial para la Península Ibérica, algunos investigadores han situado de forma tentativa en un $25 \%$ de plata y un $1 \%$ de cobre los límites considerados normales. Por encima de ellos, la composición del oro debe considerarse como una aleación artificial (Montero y Rovira 1991: 10). Según los resultados elementales de la cuenta analizada, ésta fue obtenida a partir de pepitas auríferas recolectadas en el lecho de los ríos. Los datos etnográficos apuntan como más plausible el empleo de sencillas bateas (Vázquez 1995: 158-159). Dichas pepitas suelen presentar una superficie irregular y rugosa con inclusiones de todo tipo. Debido a ello su aprovechamiento siempre requiere de una primera fase de fusión, cuyo objetivo es homogeneizar el metal y limpiarlo de impurezas. El oro resultante o bien se vacía en el interior de un molde o bien se deja solidificar en el fondo del crisol, dando como resultado un botón de fundición (Perea 2010: 248). Posteriormente, algunas piezas, como la aquí estudiada, serían trabajadas mediante batido, quizás intercalando sucesivas fases de reco-

\begin{tabular}{|l|l|c|c|}
\hline \multicolumn{1}{|c|}{ N. $^{\text {}}$ ANAL } & \multicolumn{1}{|c|}{ ZONA } & \multicolumn{1}{c|}{ Au } & \multicolumn{1}{c|}{ Ag } \\
\hline TOS01201 & Centro & 98,15 & 1,85 \\
TOS01202 & Extremo 1 & 98,26 & 1,74 \\
TOS01203 & Centro & 98,37 & 1,63 \\
TOS01205 & Extremo 2 & 98,56 & 1,44 \\
TOS01206 & Extremo 2 & 98,57 & 1,43 \\
\hline
\end{tabular}

Tab. 1. Resultados del análisis de composición (MEBEDX) efectuado en diferentes puntos de la cuenta del Cau del Tossal Gros (Torroella de Montgrí, Baix Empordà, Girona). N. ${ }^{\circ}$ Anal = Número de análisis. Valores expresados en $\%$ en peso.

cido hasta obtener una fina lámina de oro. Esta podría ser finalmente cortada, enrollada $\mathrm{y}$, en algunos casos, perforada y/o decorada. El proceso de trabajo expuesto se atestigua desde los momentos iniciales del uso de este metal en la Prehistoria, como demuestran el gran tamaño de algunas piezas peninsulares (diademas) o su ínfimo espesor (Perea 1991b: 35). Asimismo, el estudio de los espectaculares ornamentos de la necrópolis de Varna (Bulgaria), fechados a mediados del V milenio cal ANE (Ivanov 1991: 10), también apoyan esta idea.

El análisis radiológico ha puesto en evidencia que nos encontramos frente a un ornamento compuesto. La cuenta consta de dos láminas de oro independientes: cada una fue enrollada en forma troncocónica $\mathrm{y}$, posteriormente, unida con su compañera para obtener la longitud total de la pieza. Este proceso, sin paralelos peninsulares conocidos, se constata por las líneas de juntura, perpendiculares y paralelas al eje de la pieza. Las perpendiculares se localizan en el centro de la cuenta y la circundan. Estas muestran con increíble claridad la franja en la que se superponen ambas láminas, que no supera los $2 \mathrm{~mm}$, así como los extremos de las mismas. Las zonas oscuras visibles en el interior de las líneas pueden corresponder a variaciones en el espesor debido al proceso mecánico de unión de las láminas. Las dos líneas restantes, paralelas al eje de la pieza, solo se observan de forma parcial. Resultan de la superposición de los extremos de mayor longitud de cada lámina para adoptar la forma troncocónica. Es importante remarcar que cada línea sigue la misma orientación pero se encuentra en una posición diferente probando que las láminas primero fueron enrolladas y, a continuación, unidas 
entre sí para formar la cuenta bitroncocónica. El proceso inverso habría resultado en la coincidencia total entre las líneas. Todas estas junturas permanecen invisibles a simple vista. Únicamente las perpendiculares han podido ser parcialmente detectadas en el interior de la cuenta (Fig. 4).

Sucesivos análisis han confirmado la enorme similitud en la composición elemental de ambas láminas (Tab. 1). Esto apunta al empleo de una materia prima de origen similar. Las dos láminas fueron recortadas a partir de una única base o de forma independiente pero con pepitas del mismo placer. Una vez obtenidas y dobladas, la unión de los extremos de las láminas y de estas entre sí se llevó a cabo mediante ciclos alternantes de bruñido y recocido. El primer proceso generó una deformación plástica por rozamiento y presión, quizás empleando una herramienta lítica de superficie dura y lisa. El segundo fue fundamental para quitar acritud y eliminar las junturas de

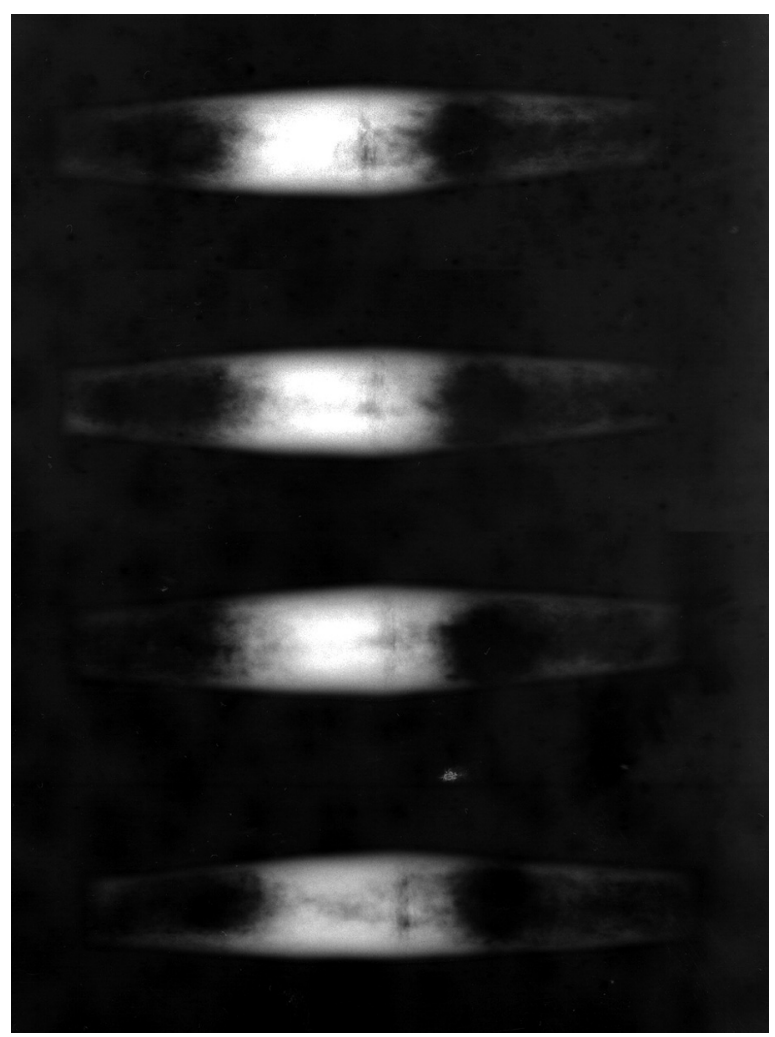

Fig. 4. Cau del Tossal Gros (Torroella de Montgrí, Baix Empordà, Girona): radiografías de la cuenta de oro, evidenciando las líneas de juntura de las dos láminas troncocónicas (fotografía Ignacio Soriano). unión así como posibles fisuras. Una simple hoguera hubiera bastado para alcanzar la temperatura necesaria. El examen traceológico no ha detectado huellas de producción vinculadas a la eliminación de las junturas en frío, ni en el interior ni en el exterior de la pieza. Este hecho confirma la técnica de unión propuesta. Un gran número de estrías se distribuyen por toda la superficie de la cuenta, mayoritariamente de forma uniforme y con idéntica orientación. Son paralelas entre sí, oblicuas al eje de la pieza, de escasa profundidad y longitud variable (Fig. 5: 1,2). Sus características las relacionan con el pulido final con un abrasivo de grano fino. Este acabado, común en las piezas áureas, tenía como objetivo resaltar el aspecto brillante de la cara externa y visible del ornamento (Perea 1991b: 36).

Otras huellas de la cuenta deben relacionarse con procesos postdeposicionales y/o tafonómicos. El origen de las estrías minoritarias y aisladas, de orientación y longitud variable hay que buscarlo en el golpeo accidental de la pieza. También se observan cuatro alineaciones formadas por sucesivas muescas perpendiculares entre sí y a distancia regular (Fig. 5: 3). Esta morfología, similar a una rodada dejada por un carro, parece vincularse a una modificación reciente. Finalmente el interior de la pieza presenta algunas estrías aisladas, paralelas a su eje y de importante longitud. Pueden deberse a una limpieza actual del orificio de la cuenta mediante un material más duro que el oro.

Los resultados obtenidos mediante la aplicación de diversas técnicas de análisis han documentado las siguientes fases del proceso de producción de la cuenta de Cau del Tossal Gros:

1. Recogida de pepitas de oro aluvial y fusión del metal. Se desconoce si la colada habría sido vaciada en un molde o se la habría dejado solidificar en el fondo de un crisol para obtener un botón de fundición.

2. Constitución de dos láminas mediante batido intercalando fases de recocido, bien de forma independiente bien recortando una única plancha inicial. El resultado final es el mismo: dos láminas de tamaño similar y morfología rectangular o ligeramente trapezoidal.

3. Enrollado de cada lámina formando dos troncos de cono separados, unión y eliminación de las líneas de juntura a través de la técnica del bruñido en conjunción con ciclos alternantes de recocido. 


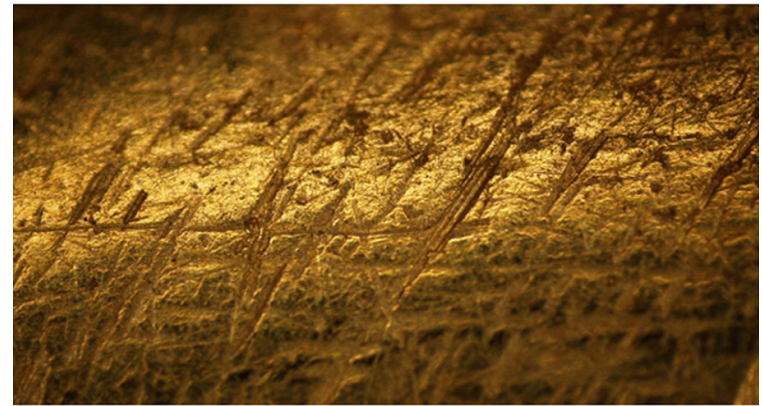

1

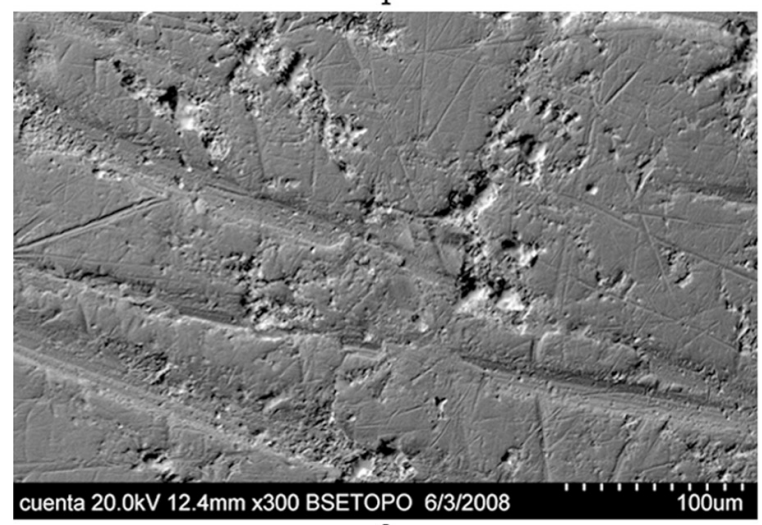

2

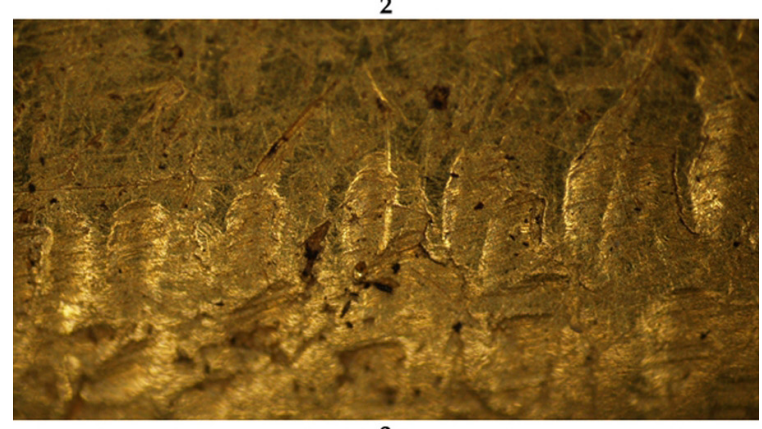

Fig. 5. Cau del Tossal Gros (Torroella de Montgrí, Baix Empordà, Girona), cuenta de oro: 1. Estrías de producción vinculadas con el pulido final de la superficie de la cuenta (microscopía óptica, 25X); 2. Detalle de las estrías mediante electrones retrodispersados (MEB-EDX, 300X); 3. Muescas postdeposicionales y/o tafonómicas de origen reciente (microscopía óptica, 25X) (fotografías 1 y 3 Ignacio Soriano, fotografía 2 Laboratorio de Microscopía Electrónica y Microanálisis, MicroLab CCHS, CSIC).

4. Unión de las dos piezas troncocónicas por bruñido y recocido para obtener la longitud total de la cuenta. No se puede o no interesa la eliminación de la línea de juntura interna.

5. Acabado y abrillantado de la superficie externa mediante pulido empleando un material abrasivo, siguiendo siempre una orientación oblicua.

\section{CRONOLOGÍA RELATIVA Y ABSOLUTA}

La necrópolis de Cau del Tossal Gros cuenta con 3 dataciones radiocarbónicas AMS realizadas en el laboratorio Beta Analytic sobre restos humanos: un fragmento de parietal (Beta - 252115) y dos occipitales (Beta - 301152 y 301153) (Tab. 2). Las fechas revelan un uso funerario prolongado de la cavidad entre c. 3450 - $2200 \mathrm{cal}$ ANE. Ello equivale, según la periodización actual, al principio del Neolítico Final, al grupo campaniforme y quizás también a un momento incipiente de la Edad del Bronce (Bronce Inicial) (Soriano 2010: 88-100). Estos resultados coinciden con la cronología relativa de los diferentes materiales recuperados en el yacimiento. Sin embargo, la fuerte alteración ya señalada de la estratigrafía de la cueva imposibilita asociar las dataciones obtenidas y la cuenta áurea.

Hasta ahora no se conocían ejemplares de este tipo de cuentas en la Península Ibérica. Los paralelos más cercanos hay que buscarlos en yacimientos funerarios del sur de Francia, todos sin dataciones absolutas (Fig. 7). Las cuentas más similares en dimensiones y morfología provienen de la región de Midi-Pyrénées. De la sepultura de Pauilhac (Gers) proceden 8, una de las cuales desapareció durante la excavación. El resto de artefactos, entre los que destaca una excepcional diadema losángica de oro, se asocian inequívocamente con el Neolítico Final. La reciente revisión de este contexto lo ha fechado de forma relativa en la primera mitad del IV milenio cal ANE (Roussot-Larroque 2008: 135). La cuenta recuperada en el dolmen de Pouy-Mayou (Bartrès, HautesPyrénées) únicamente iba acompañada de un cuchillo de sílex. Con un contexto menos claro en la región de Provence-Alpes-Côte d'Azur encontramos la procedente del hipogeo de Castellet (Fontvieille, Bouches-du-Rhône), con materiales del Neolítico Final y del grupo campaniforme (Fig. 6).

Existe otro conjunto de cuentas de morfología muy similar a las anteriores pero de tamaño tres o cuatro veces menor. Todas se sitúan en la región de Languedoc-Roussillon, documentándose una en cada yacimiento (Fig. 6: 5, 6 y 7). El dolmen de Sauzet 1 (Cazevieille) y el túmulo de Les Avents 1 (Sant-Mathieu-de-Tréviers), ambos en Hérault, presentan exclusivamente materiales de finales del Neolítico. Otra cuenta se encontró en 


\begin{tabular}{|c|c|c|c|c|c|c|c|}
\hline YACIMIENTO & $\begin{array}{c}\text { OBJ. MET. } \\
\text { ASOCIADO }\end{array}$ & $\begin{array}{c}\text { CÓDIGO } \\
\text { LAB }\end{array}$ & $\begin{array}{c}\text { VALOR } \\
\text { BP }\end{array}$ & $\begin{array}{l}\text { VALOR } \\
\text { CAL ANE }\end{array}$ & MUESTRA & CONTEXTO & BIBLIOGRAFÍA \\
\hline Tossal Gros & $\begin{array}{c}\text { Cuenta } \\
\text { biconvexa oro }\end{array}$ & Beta-252115 & $4630 \pm 40$ & 3446 & Hueso humano & - & Inédito \\
\hline Tossal Gros & $\begin{array}{c}\text { Cuenta } \\
\text { biconvexa oro }\end{array}$ & Beta-301152 & $3930 \pm 30$ & 2416 & Hueso humano & - & Inédito \\
\hline Tossal Gros & $\begin{array}{c}\text { Cuenta } \\
\text { biconvexa oro }\end{array}$ & Beta-301153 & $3790 \pm 30$ & 2223 & Hueso humano & - & Inédito \\
\hline La Prunera & $\begin{array}{c}\text { Cuenta } \\
\text { tubular oro }\end{array}$ & Beta-144301 & $4360 \pm 80$ & 2994 & Carbón & Nivel 2 & $\begin{array}{l}\text { Rovira Hortalà et al. } \\
2005\end{array}$ \\
\hline $\begin{array}{c}\text { Balma } \\
\text { de Cal Porta }\end{array}$ & $\begin{array}{c}\text { Punzón } \\
\text { cobre }\end{array}$ & UBAR-288 & $4160 \pm 60$ & 2766 & Hueso humano & $\begin{array}{c}\text { Fondo de la } \\
\text { cavidad }\end{array}$ & Estany y Guerrero 1992 \\
\hline $\begin{array}{c}\text { Cova } \\
\text { de Can Sadurní }\end{array}$ & $\begin{array}{c}\text { Puñal } \\
\text { cobre/bronce }\end{array}$ & I-11533 & $4225 \pm 90$ & 2786 & Hueso humano & Estrato I, capa 9 & Blasco et al. 1981-1982 \\
\hline $\begin{array}{c}\text { Cova } \\
\text { de Can Sadurní }\end{array}$ & $\begin{array}{c}\text { Puñal } \\
\text { cobre/bronce }\end{array}$ & I-13315 & $4139 \pm 110$ & 2737 & Carbón & Estrato I, capa 9 & Blasco et al. 1981-1982 \\
\hline $\begin{array}{c}\text { Cova } \\
\text { de Can Sadurní }\end{array}$ & $\begin{array}{c}\text { Puñal } \\
\text { cobre/bronce }\end{array}$ & I-13313 & $4160 \pm 160$ & 2712 & Carbón & $\begin{array}{c}\text { Estrato I, capa } 9, \\
\text { hogar } 2\end{array}$ & Blasco et al. 1981-1982 \\
\hline $\begin{array}{c}\text { Cova } \\
\text { de Can Sadurní }\end{array}$ & $\begin{array}{c}\text { Puñal } \\
\text { cobre/bronce }\end{array}$ & I-12717 & $4080 \pm 100$ & 2661 & Carbón & $\begin{array}{c}\text { Estrato I, capa } 9, \\
\text { hogar } 2\end{array}$ & Blasco et al. 1981-1982 \\
\hline
\end{tabular}

Tab. 2. Dataciones absolutas relacionadas con los primeros objetos de oro y de cobre del nordeste de la Península Ibérica (programa empleado Calib 5.0). El valor calibrado es el resultado de la media de la datación calibrada a $1 \sigma$ ponderada por cada uno de los rangos de valores probables existentes (Soriano 2010: 49-50). Tossal Gros (Torroella de Montgrí, Baix Empordà, Girona); La Prunera (Sant Joan les Fonts, Garrotxa, Girona); Balma de Cal Porta (Torà, Segarra, Lleida); Cova de Can Sadurní (Begues, Baix Llobregat, Barcelona). Obj. met. = objetos metálicos; Lab. = Laboratorio.

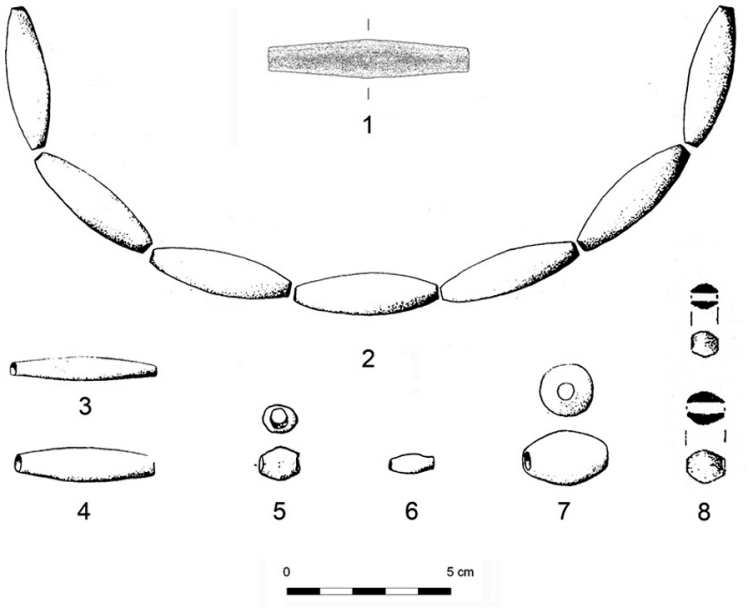

Fig. 6. Cuenta bitroncocónica de oro del Cau del Tossal Gros (Torroella de Montgrí, Baix Empordà, Girona) (n. ${ }^{\circ}$ 1) y ejemplares citados del sur de Francia y Portugal: 2. Pauilhac (Gers, Midi-Pyrénées); 3. Pouy-Mayou (Bartrès, Hautes-Pyrénées, Midi-Pyrénées); 4. Castellet (Fontvieille, Bouches-du-Rhône, Provence-Alpes-Côted'Azur); 5. Sauzet 1 (Cazevieille, Hérault), 6. Les Avents 1 (SantMathieu-de-Tréviers, Hérault) y 7. Saint-Eugène (Laure, Aude) en Languedoc-Rousillon; 8. Buraco da Pala (Mirandella, Portugal). 2 a 7 según Eluère (1977: 392), 8 según Comendador (1998: 218).
Saint-Eugène (Laure, Aude) acompañada de artefactos vinculados al grupo campaniforme. Por último, el hallazgo de la cueva de Porte (Narbonne, Aude) se asocia sin ninguna duda al Bronce Antiguo, constituyendo la única perduración detectada en esta cronología (Eluère 1982: 27).

Los únicos objetos áureos ligeramente similares al de Cau del Tossal Gros en la Península Ibérica proceden del abrigo de Buraco da Pala (Mirandella, Portugal). Son 2 cuentas bitroncocónicas de pequeñas dimensiones fechadas por radiocarbono a mediados del III milenio cal ANE (Fig. 6: 8). Fueron localizadas en el nivel 1 de la cavidad, asociadas a 4 cuentas esféricas y una lámina de oro así como a varias concentraciones de cuentas de piedra, mayoritariamente variscita (Comendador 1998: 110-111).

Según Eluère (1977: 407-408; 1982: 136) la cronología relativa de las cuentas galas se sitúa claramente en el Neolítico Final, tal y como atestiguan varios contextos carentes de materiales más modernos (Pauilhac, Pouy-Mayou, Sauzet 1, Les Avents 1). La reutilización de estas sepulturas por el grupo campaniforme o incluso alguna perduración durante el II milenio cal ANE no cues- 


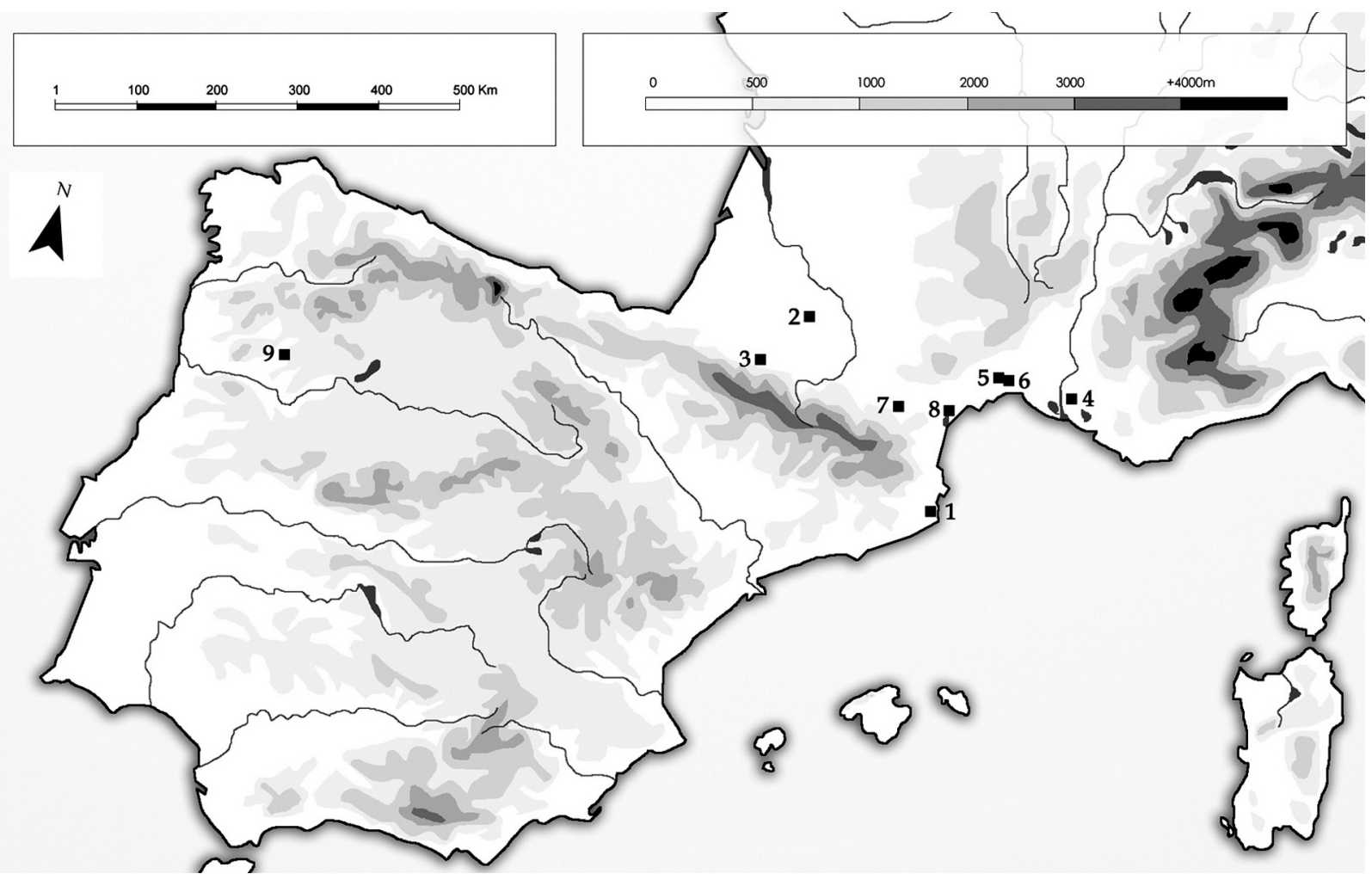

Fig. 7. Localización según el orden enumerado en el texto de las cuentas bitroncocónicas galas y portuguesas de oro citadas como paralelos de la 1. Cau del Tossal Gros (Torroella de Montgrí, Baix Empordà, Girona): 2. Pauilhac (Gers, Midi-Pyrénées); 3. Pouy-Mayou (Bartrès, Hautes-Pyrénées, Midi-Pyrénées); 4. Castellet (Fontvieille, Bouches-du-Rhône, Provence-Alpes-Côte d'Azur); 5. Sauzet 1 (Cazevieille, Hérault), 6. Les Avents 1 (Sant-Mathieu-de-Tréviers, Hérault), 7. Saint-Eugène (Laure, Aude) y 8. Cueva de Porte (Narbonne, Aude) en Languedoc-Rousillon; 9. Buraco da Pala (Mirandella, Portugal).

tionan esta afirmación. Los dos casos de Buraco da Pala corroboran esta cronología antigua. Por otra parte, la ausencia de cuentas análogas en el resto de territorio europeo con presencia campaniforme refuerza la desvinculación entre ambos fenómenos.

El conjunto de datos expuestos nos inclina a pensar que la cronología de la cuenta de Cau del Tossal Gros no debe alejarse demasiado de finales del IV-inicios del III milenio cal ANE. Así lo indican la cronología relativa de los casos franceses y la proximidad geográfica con los mismos. La datación radiocarbónica más antigua obtenida en el yacimiento catalán (Beta - $252115=3450$ cal ANE), aún sin poder asociarse con la cuenta, es coherente con esta afirmación. A la vez se constata que este tipo de adornos se vinculan inequívocamente con el momento inicial de los grupos del Neolítico Final.

\section{CONCLUSIONES. LA PRIMERA ORFEBRERÍA DEL NEOLÍTICO FINAL}

El estudio de la cuenta de oro de Cau del Tossal Gros ha documentado uno de los ejemplos más antiguos de orfebrería prehistórica del nordeste de la Península Ibérica. A la vez y con cierta sorpresa, la tecnología empleada en su producción (unión por bruñido de dos piezas laminares) denota un elevado grado de conocimiento y destreza técnica. No tenemos constancia de la existencia de objetos con similares características en el resto de la Península Ibérica en fechas tan tempranas.

En el área catalana los primeros objetos áureos se sitúan dentro del Neolítico Final, en torno al 3000 cal ANE. Son los objetos de metal más antiguos puesto que los primeros artefactos de cobre se fechan en momentos ligeramente poste- 
riores (c. 2800 cal ANE) (Tab. 2). Las 4 únicas piezas de oro documentadas en todo el territorio son elementos ornamentales. De Balma dels Ossos (Berga, Berguedà) y Cabana Arqueta (Espo1la, Alt Empordà) provienen 2 cuentas de tipo esférico, carentes todavía de estudio tecnológico que determine su proceso de producción (Castillo 1962; Tarrús 2002: 311). La única cuenta tubular conocida, recuperada en La Prunera (Sant Joan les Fonts, Garrotxa), consiste en una fina lámina rectangular enrollada sobre sí misma (Rovira Hortalà et al. 2005). Los objetos de oro vinculados con el grupo campaniforme (c. 2800 - 2350 cal ANE) son más abundantes. Son cuentas tubulares y apliques de lados opuestos abatidos procedentes de yacimientos del Empordà: Solar d'en Gibert, Cova d'en Daina, Barranc o Dolmen d'en Coto y Puig Roig o Cementiri dels Moros (Esteva 1964, 1970, 1976; Tarrús et al. 1983: 38-40; Tarrús 2002: 343-356, 381-388). Sin embargo, tampoco estas denotan una tecnología que supere el trabajo de batido y recortado de un único producto laminar (Soriano 2010: 302-310). ¿Guardan estas diferencias tecnológicas alguna relación con el origen de la orfebrería en esta región?

Actualmente, ninguna evidencia demuestra la producción de objetos de oro entre los grupos del Neolítico Final. Ciertamente, las labores de orfebrería requieren herramientas poco especializadas, muy similares a las empleadas en la metalurgia de base cobre (martillos, yunques, pulidores) (Eluère 1982: 206; Armbruster 2010: 14-17). Sin embargo, tampoco existen testimonios de la producción de cobre en estos grupos. Los vasos de reducción y restos de fundición más antiguos se relacionan inequívocamente con el grupo campaniforme (Balma del Serrat del Pont, Cova del Frare, Vapor Gorina) (Martín et al. 1985; Alcalde et al. 1998; Roig et al. 2009). A ello cabe sumar, como se ha visto, que los primeros objetos de cobre son ligeramente más tardíos que los de oro. En sus inicios ambos metales sirvieron únicamente como productos manufacturados, siendo desconocido su proceso de producción. Los datos cronológicos, tipológicos y culturales atestiguan la vinculación entre estos primeros objetos y los centros metalúrgicos del sur de Francia (Soriano 2010: 486-497). Las continuas interacciones entre ambas regiones, visibles también en otras evidencias arqueológicas, denotan un constante movimiento de sujetos y objetos. La materialidad arqueológica mayoritaria del Neolítico Final del nordeste de la Península Ibérica ha sido definida hasta día de hoy como Véraza, debido a las importantes similitudes que presenta con el grupo homólogo francés del que se supone parte integrante (Martín 2003: 83-84). Otros artefactos documentados de forma más limitada, como cerámicas decoradas, cuentas de aletas globulares o un botón de caliza tipo Durfort han sido vinculados a otros grupos de la misma región (Treïlles, Ferrières, Fontbouisse) (Martín et al. 2002). Más allá de estas evidencias materiales, determinadas prácticas económicas e ideológico-simbólicas apuntan también hacia el otro lado de los Pirineos. La reciente documentación de varias estatuas-menhir antropomorfas y grandes estructuras de combustión con piedras termoalteradas tienen, en el sur de Francia, unos referentes innegables (Fortó et al. 2008; Moya et al. 2010). "Los paralelos expuestos en la cuenta de Cau del Tossal, procedentes en su totalidad del país galo, constituyen una prueba fehaciente". La ausencia de análisis similares en las piezas francesas impide conocer si repiten el característico proceso de producción documentado en la cuenta catalana. De lo que no cabe ninguna duda es que semejante procedimiento tecnológico parece ser desconocido en la Península Ibérica de forma sincrónica (Perea 1991b: 35-36; Perea et al. 2010: 15-19).

Un segundo aspecto a señalar se refiere al papel que el metal y, en concreto, el oro tuvo en el seno de estas comunidades. La morfología de las cuentas muestra una enorme coincidencia con los tipos en uso contemporáneamente sobre otros soportes (líticos, óseos, malacológicos). Se documentan cuentas esféricas realizadas sobre diferentes rocas y resinas fósiles. Las bitroncocónicas guardan una gran semejanza con las más antiguas de variscita y las cuentas tubulares con los dentalia. El oro se emplea inicialmente para producir ornamentos análogos a los ya existentes en vez de nuevos. Este dato indica que el aprovechamiento del metal no puede relacionarse con sus mejores propiedades físicas y mecánicas (ductilidad, moldeado, reciclado) respecto a las demás materias. El conocimiento profundo de dichas propiedades conllevaría el desarrollo de artefactos mejor adaptados al metal, como ocurre a partir de la irrupción del grupo campaniforme. Únicamente aspectos como la capacidad de reflejar la luz, que le aporta su peculiar brillo dorado, pueden explicar en parte su aceptación. A ello hay que sumar que los ornamentos de oro son cuantitativamente anecdó- 
ticos dentro del registro arqueológico de este momento, caracterizado por una gran diversidad de elementos decorativos. Todo ello indica que el oro sería más bien un objeto de curiosidad, que se emplearía conjuntamente con otras cuentas no metálicas formando parte del mismo atavío. Su repercusión social sería muy limitada y su valor social no se alejaría, pues, excesivamente del otorgado a los restantes ornamentos.

Finalmente, se constata que los contextos de hallazgo son funerarios salvo un caso en hábitat (La Prunera). Este hecho, documentado de forma idéntica en la mitad meridional de la Península Ibérica, apunta hacia un uso del oro no exclusivamente funerario (Perea 1991a: 298, 1991b: 273275). Los elementos decorativos áureos se emplearían, como los restantes, en la vida diaria. Su morfología relativamente robusta aseguraría cierta resistencia y durabilidad, visible en la cuenta de Cau del Tossal Gros. La introducción de muchas de ellas en las tumbas, siempre de tipo múltiple colectivo, responde a la inhumación del individuo con sus abalorios cotidianos y no a la deposición de elementos producidos expresamente con fines funerarios (Soriano 2010: 315-316). Todo lo contrario se constata a partir del $2800 \mathrm{cal}$ ANE con la irrupción del grupo campaniforme. Por vez primera aparecen nuevas morfologías de ornamentos, con una estructura más frágil (apliques de lados abatidos) procedentes exclusivamente de tumbas claramente individualizadas (Soriano 2010: 316318). Algunos de estos notables cambios en el uso del oro han sido igualmente documentados en áreas cercanas, como el Alto Valle del Ebro (Rodríguez de la Esperanza 2004, 2005: 105-108) o el sur de Francia (Eluère 1982: 197). El gran salto social desde el uso del producto metálico a la producción metalúrgica había empezado.

\section{AGRADECIMIENTOS}

Por su colaboración y soporte técnico a los siguientes investigadores: Alicia Perea (Centro de Ciencias Humanas y Sociales, Consejo Superior de Investigaciones Científicas, Madrid), José M. ${ }^{\mathrm{a}}$ González y Jesús Serrano (Asociación Española de Ensayos No Destructivos, Madrid) y Elio Vivas y Rosa Dinares (Sección de Radiología y Angiografía Rotacional del Hospital General de Catalunya). Toni Roviras, director del Museo del Mediterráneo, nos dio todas las facilidades posi- bles para el estudio de la cuenta. Al resto de miembros de Grupo de Investigación Arqueológica del Nordeste Peninsular - GRANEP, Paz Balaguer, Emiliano Hinojo y Camila Oliart así como a los dos evaluadores anónimos por la revisión y comentarios a este artículo. Esta investigación ha sido financiada por la Fundación Juanelo Turriano (Beca de Doctorado en Historia de la Ciencia y de la Técnica) y la Secretaría de Universidades del Departamento de Economía y Conocimiento de la Generalitat de Catalunya (2010ACOM00047).

\section{BIBLIOGRAFÍA}

Agustí, B. y Fiego, J. 2002: "Estudi antropològic de la cova sepulcral del Tossal Gros (Torroella de Montgrí)". XII Col-loqui Internacional d'Arqueologia de Puigcerdà (Puigcerdà 2000): 723-728. Puigcerdà.

Alcalde, G.; Molist, M.; Montero, I.; Planagumà, Ll.; Saña, M. ${ }^{a}$ y Toledo, A. 1998: "Producciones metalúrgicas en el nordeste de la Península Ibérica durante el III milenio cal. AC: El taller de la Bauma del Serrat del Pont (Tortellà, Girona)". Trabajos de Prehistoria 55 (1): 81-100.

Armbruster, B. 2010: "Lithic technology for Bronze Age metalworking". En B. Valentin (ed.): Lithic technology in metal using societies. Proceedings of a UISPP Workshop (Lisbon 2006): 9-22. Højbjerg.

Blasco, A.; Edo, M.; Blanch, M. y Millán, M. 19811982: "La Cova de Can Sadurní, una cruilla de camins". Pyrenae 17-18: 11-34.

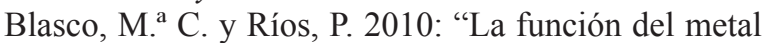
entre los grupos campaniformes. Oro versus cobre. El ejemplo de la Región de Madrid". Trabajos de Prehistoria 67 (2): 359-372.

Castillo, A. 1962: "La Balma dels Ossos de Berga". VII Congreso Nacional de Arqueología (Barcelona 1960): 201-208. Zaragoza.

Comendador, B. 1998: "Los inicios de la metalurgia en el Noroeste de la Península Ibérica”. Brigantium 11.

Eluère, Ch. 1977: "Les premiers Ors en France". Bulletin de la Société Préhistorique Française 74: 390-419.

Eluère, Ch. 1982: Les ors préhistoriques. L'Age du Bronze en France 2. Picard. París.

Estany, I. y Guerrero, Ll. 1992: Memòria de l'excavació d'urgència a la Balma de Cal Porta a Torà (La Segarra) 1990-1991. Servei d'Arqueologia i Paleontologia, Generalitat de Catalunya. Barcelona.

Esteva, L1. 1964: Sepulcros megalíticos de las Gabarras (Gerona) I. Corpus de Sepulcros Megalíticos 3. CSIC. Gerona. 
Esteva, Ll. 1970: Sepulcros megalíticos de las Gabarras (Gerona) III. Corpus de Sepulcros Megalíticos 5. CSIC. Gerona.

Esteva, L1. 1976: "Sepulcros megalíticos de las Gabarras. Noticias complementarias". Cypsela, 2: 55-89.

Fortó, A.; Martínez, P. y Muñoz, V. 2008: "Las estructuras de combustión de grandes dimensiones de Ca l'Estrada en el neolítico europeo". En M. S. Hernández, J. A. Soler y J. A. López Padilla (eds.): Actas del IV Congreso de Neolítico Peninsular (Alicante 2006): 306-314. Alicante.

Ivanov, I. S. 1991: "Les objets métalliques de la nécropole chalcolithique de Varna". En J. P. Mohen y C. Éluère (eds.): Découverte du métal. Millénaires 2, Picard. París: 9-11.

Martín, A. 2003: "Els grups del neolític final, calcolític i bronze antic. Els inicis de la metal-lúrgia". Cota Zero, 18: 76-105.

Martín, A.; Biosca, A. y Albareda, M. J. 1985: "Excavacions a la Cova del Frare (Matadepera, Vallès Occidental). Dinàmica ecològica, seqüència cultural i cronologia absoluta". Tribuna d'Arqueologia 1983-1984: 91-103.

Martín, A.; Petit, M. ${ }^{a}$ A. y Maya, J. L. 2002: "Cultura material, economia i intercanvis durant el III mil-lenni a Catalunya". XII Col-loqui Internacional d'Arqueologia de Puigcerdà (Puigcerdà 2000): 295-321. Puigcerdà.

Montero, I. y Rovira, S. 1991: "El oro y sus aleaciones en la orfebrería prerromana". Archivo Español de Arqueología, 64: 7-21.

Moya, A.; Martínez, P. y López, J. B. 2010: "Éssers de pedra. Les estàtues-menhirs i esteles antropomorfes de l'art megalític de Catalunya". Cypsela 18: 11-41.

Pascual, J. 1883: "Una excursión a la cueva funeraria de Torroella de Montgrí". El Demócrata, Gerona, 11 de noviembre.

Perea, A. 1991a: "L'apparition de la métallurgie de l'or dans la moitié méridionale de la Péninsule Ibérique. Problèmes et méthodologie". En J. P. Mohen y C. Éluère (ed.): Découverte du métal. Millénaires 2, Picard. París: 295-302.

Perea, A. 1991b: Orfebrería prerromana. Arqueología del Oro. Caja Madrid y Comunidad de Madrid. Madrid.

Perea, A. 2010: "Arqueología del oro: Tecnología de los metales nobles". En I. Montero (coord.): $\mathrm{Ma}$ nual de Arqueometalurgia. Cursos de formación permanente para arqueólogos, Museo Arqueológico Regional de la Comunidad de Madrid. Alcalá de Henares: 233-267.

Perea, A.; García Vuelta, O. y Fernández Freire, C. 2010: El Proyecto Au. Estudio arqueométrico de la producción de oro en la Península Ibérica. Bibliotheca Praehistorica Hispana XXVII, CSIC. Madrid.
Pericot, L. 1925: La civilización megalítica catalana y la cultura pirenaica. Universidad de Barcelona. Barcelona.

Pericot, L. 1939: "Las cuevas sepulcrales del Montgrí". Ampurias, 1: 113-137.

Pericot, L. 1950: La civilización megalítica catalana y la cultura pirenaica. CSIC, Instituto de Estudios Pirenaicos. Barcelona, 1. ${ }^{a}$ ed. 1925.

Pericot, L. 1960: "Cuevas prehistóricas del Montgrí. El Cau de l'Olivar d'en Margall". Llibre de la Festa Major de Torroella de Montgrí. Associació del llibre de la festa major. Torroella de Montgrí http://www.raco.cat/index.php/LlibreFestaMajor/ article/view/210209/279434 (consulta 7-III-2012).

Pericot, J. 1986: "Un record de Pere Bosch i Gimpera des de Torroella". Llibre de la Festa Major de Torroella de Montgrí. Associació del llibre de la festa major. Torroella de Montgrí: 1-5.

Pericot, L. y Esteva, L. 1973: "La cueva-dolmen del Tossal Gros (Torroella de Montgrí, Gerona)". XII Congreso Nacional de Arqueología (Jaén 1971): 191-200. Zaragoza.

Rodríguez de la Esperanza, M.a J. 2004: "La primera industria del oro en el Valle del Ebro". En A. Perea, I. Montero y O. García-Vuelta (eds.): Tecnología del oro antiguo: Europa y América. Anejos de Archivo Español de Arqueología XXXII, CSIC. Madrid: 189-195.

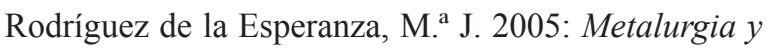
metalúrgicos en el Valle del Ebro (c. 2900-1500 cal A.C.). Bibliotheca Archaeologica Hispana 24, Real Academia de la Historia, Institución "Fernando el Católico". Madrid.

Roig, J.; Molina, D.; Coll, J. M. y Molina, J. A. 2009: "El jaciment calcolític del Vapor Gorina (Sabadell, Vallès Occidental)". Tribuna d'Arqueologia 2007: 93-122.

Roussot-Larroque, J. 2008: "La 'sépulture de chef' de Pauilhac (Gers)". Préhistoire du Sud-Ouest 16, 1: 91-142.

Rovira Hortalà, M. ${ }^{a}$ C.; Borrell, F.; Oliva, M.; Saña, M. a Vicente, O. y Casellas, S. 2005: "Las primeras manufacturas de oro en Catalunya: nuevos datos procedentes del yacimiento de La Prunera (Girona)". En O. Puche y M. Ayarzagüena (eds.): Minería y Metalurgia históricas en el Sudoeste Europeo (Madrid 2004). Sociedad Española para la Defensa del Patrimonio Geológico y Minero. Madrid: 53-60.

Soler, N.; Roviras, A.; Soler, J.; Casas, S.; Maragall, Ll. y Ramió, S. 2002: "El Cau del Tossal Gros: una cavitat paradolmènica del massís del Montgrí". XII. ${ }^{\circ}$ Col-loqui Internacional d'Arqueologia de Puigcerdà (Puigcerdà 2000): 709-722. Puigcerdà.

Soriano, I. 2010: Producción metalúrgica prehistórica en el nordeste de la Península Ibérica (mediados del IV-II milenio cal ANE). Aportaciones cronoculturales, tecnológicas y funcionales. Tesis Doctoral, 
2 vols. + CD Rom, Universidad Autónoma de Barcelona. Barcelona.

Tarrús, J. 2002: Poblats, dòlmens i menhirs els grups megalítics de l'Albera, serra de Rodes i cap de Creus (Alt Empordà, Rosselló $i$ Vallespir Oriental). Diputació de Girona. Girona.

Tarrús, J.; Castells, J.; Vilardell, R. y Chinchilla, J. 1983: Els dolmens de Comes Llobes de Pils $i$ de Solar d'en Gibert. Excavacions Arqueològiques a Catalunya 4, Generalitat de Catalunya, Barcelona.

Toledo i Mur, A.; Rueda i Torres, J. M.; Vila i Mitja, A.; Merino Nazábal, I.; Agustí i Farjas, B. y
Pons i Bruns, E. 1992: El Cau d'En Calvet. Un enterrament del Neolític. Papers del Montgrí 9, Museu del Montgrí i del Baix Ter. Torroella de Montgrí.

Vázquez, J. M. 1995: "Etnoarqueología de la extracción del oro de los ríos en el Noroeste de la Península Ibérica". Trabajos de Prehistoria 52 (2): 157-161.

Vert, J. 1980: "Noves troballes arqueològiques a la 'cova dolmènica' del Tossal Gros (Montgrí-Baix Empordà)". Butlletí de l'Associació Arqueologica de Girona 3: 34-35. 